See discussions, stats, and author profiles for this publication at: https://www.researchgate.net/publication/251066674

\title{
The Global Ageing Experience Project: Anthropology, technology and
} independent living

Article in Gerontechnology · April 2010

DOl: $10.4017 /$ gt.2010.09.02.275.00

CITATIONS

4 authors, including:

David Prendergast

National University of Ireland, Maynooth

47 PUBLICATIONS 389 CITATIONS

SEE PROFILE

Some of the authors of this publication are also working on these related projects:

Project Intel Digital Health Research View project

Project Smart Cities View project

\section{READS}

85

John Sherry

Intel

26 PUBLICATIONS 217 CITATIONS

SEE PROFILE 
T. Plowman, D. Prendergast, S. Roberts, J. Sherry. The Global Ageing Experience Project: Anthropology, technology and independent living. Gerontechnology 2010;9(2):243; doi:10.4017/gt.2010.09.02.275.00 Purpose Adoption of a range of assistive technologies among individuals who desire to age in place or otherwise live independently has been sporadic and uneven over the past decade, never reaching significant adoption rates except in a few instances. There are a number of possible reasons for this: lack of a board reimbursement structure, narrow or poor marketing, cost, niche products, to name a few. Another plausible reason is that in the planning and ultimate design of these systems and technologies, too little attentions has been paid to the cultural and experiential dimensions of aging. Aging is a social as well as biological process and insights into the experience of aging that can reflected in the design of gerontechnological systems will only support adoption. In 2006 anthropologists from Intel's Digital Health Group launched a multi-year, multi-sited ethnographic research project designed to develop a global, comparative understanding of the practices and meanings associated with the later life course with the ultimate goal of identifying ways in which technology might play a role in supporting 'healthy aging'. Previous work in this area, while useful, frequently stops short of applying insights to technologically oriented solutions ${ }^{1,2}$. Approaching the challenge of designing gerontechnology from a human factors perspective has fared no better over the past decade, frequently paring back the experience of a system or technology to a series of narrowly described and atomized tasks distinct from their socio-cultural context ${ }^{3}$. Method The first leg of this project saw research conducted in 75 older households in seven European countries. The sample includes older people with physical and cognitive impairments, those living with life-long or chronic diseases, 'healthy' older people as well as many additional interviews with informal caregivers and healthcare experts from each nation. The qualitative data was supplemented by detailed institutional and policy oriented research reports focused on aging in each region. Results \& Discussion Analysis led to the identification of major tropes constituting the experience of aging that potentially align with applications of information and communications technology. In this paper we describe some of the people we've met, as a way of introducing key findings in our ethnographic work, and how they have shaped the questions we ask as we imagine new technologies of independent living for the future.

\section{References}

1. Sokolovsky J, editor. The Cultural Context of Aging; Worldwide Perspectives. Santa

BArbara: Greenwood

Press;2008

2. Katz S. Disciplining Old Age: The Formation of Gerontological Knowledge. Charlottesville: University of Virginia Press; 1996

3. Rogers W, editor. Designing for an Aging Population : Ten Years of Human Factors/Ergonomics Research. Santa Monica: Human Factors and Ergonomics Society; 1997

Keywords: cultural, comparative, ethnography

Address: Intel Corporation, USA;

E: tim.plowman@intel.com

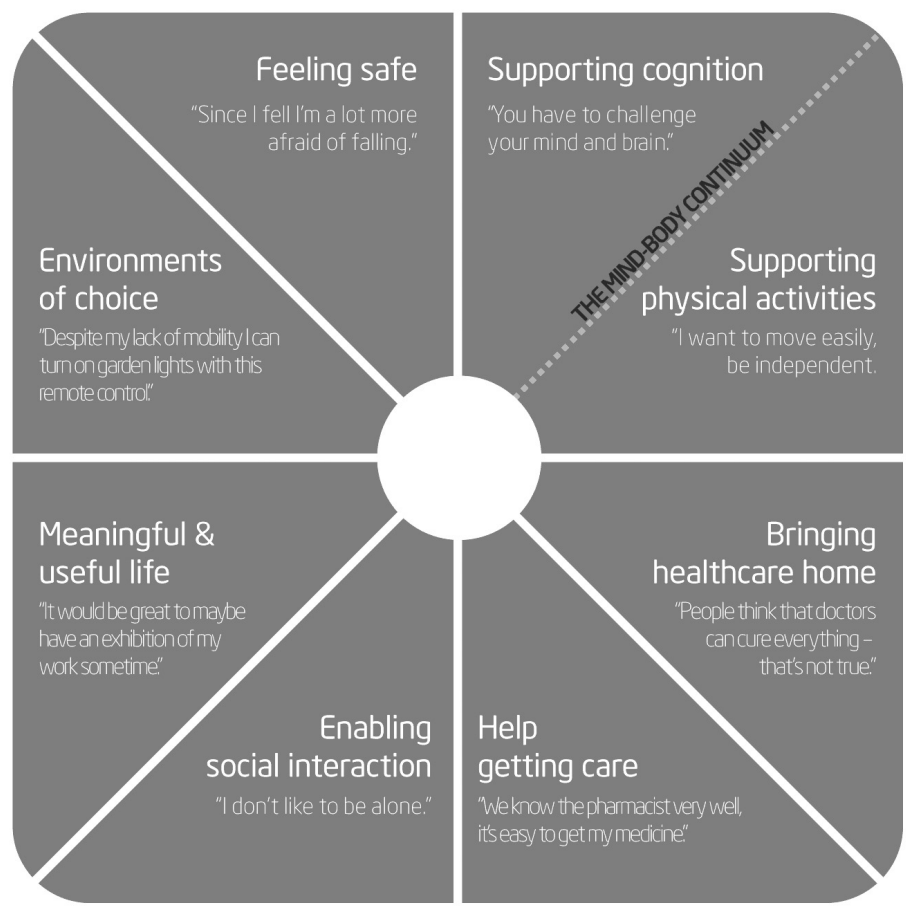

Figure 1: Outline of 8 contexts in which key themes of the aging experience create opportunities for the nuanced application of gerontechnology across cultures 